\title{
Elgar framework: context-aware service orchestration with data Petri net
}

\begin{abstract}
The Internet of Things is composed of many heterogeneous devices and services. In general, the phase of orchestrating different devices in order to allow interoperability in different environment is difficult. This is because most IoT services are not reusable because of data interpretation and service interoperability problem. In this research, we embrace the concept of design once, deploy anywhere for IoT services. We proposed two major methods which are (i) modeling IoT services with data-aware service model and (ii) semantical approach using context ontology to support service orchestration. Finally, we showed that IoT services with various ontologies can be composed based on our orchestration method.
\end{abstract}

Keyword: IoT; Elgar framework 